\title{
Simplified local anesthesia technique for external dacryocystorhinostomy without nasal packing: a new technique and pilot study outcome
}

This article was published in the following Dove Press journal:

Clinical Ophthalmology

26 November 2013

Number of times this article has been viewed

Hatem A Tawfik'

Osama R Youssef ${ }^{2}$

'Department of Ophthalmology, ${ }^{2}$ Department of Anesthesiology, Ain Shams University, Cairo, Egypt

Correspondence: Hatem A Tawfik Ain Shams University, 5 lbn Elnafis St, Off Makram Ebeid St, Nasr City, Cairo, Egypt $\mathrm{Tel}+20$ I0I 0225 I 3

Fax +2022749374

Email hatem35@gmail.com
Background: The purpose of this paper is to describe a simplified local anesthesia technique for external dacryocystorhinostomy (EXT-DCR).

Methods: In this pilot, retrospective, noncomparative, interventional case series, 448 patients (480 eyes) underwent EXT-DCR using a simplified local anesthesia technique. Nasal mucosal anesthesia was achieved using combined application of $6 \mathrm{~mL}$ of oxymetazoline $0.025 \%$ nasal spray and lidocaine $1 \%$ in the same spray bottle, without any packing of the nose with either pledgets or ribbon gauze. Local infiltration anesthesia consisted of subcutaneous injection of a $7 \mathrm{~mL}$ mixture of 2\% lidocaine with 1:100,000 epinephrine injected on the flat side of the nose beneath the incision site, in addition to a second medial peribulbar injection $(3 \mathrm{~mL}, 2 \%$ lidocaine without epinephrine).

Results: Successful unilateral or bilateral EXT-DCR was achieved in 432/448 patients (96.4\%). Four patients could not tolerate the procedure under local anesthesia and were converted to general anesthesia. Four patients required additional local anesthetic injections because of intolerable pain. Heavy sedation was essential in eight uncooperative patients because surgical manipulation was impossible. The remaining patients tolerated the procedure well. The intraoperative bleeding rate was very low except in one patient. Mean operative time was 16 minutes. Severe postoperative epistaxis was observed in four patients. Temporary anosmia developed in one patient.

Conclusion: Our simplified local anesthesia approach of EXT-DCR is convenient for the patient because it avoids unnecessary nasal packing. It is also safe and effective, as evidenced by the high rate of successful completion of the procedure without conversion to general anesthesia or the need for supplemental local anesthesia.

Keywords: local anesthesia, external dacryocystorhinostomy

\section{Introduction}

External dacryocystorhinostomy (EXT-DCR) is still considered the treatment of choice for postsaccal nasolacrimal duct obstruction. ${ }^{1,2}$ Currently, in our practice, we offer our patients the choice to perform the procedure either transcutaneously (EXT-DCR) under local anesthesia, or endoscopically (END-DCR) under general anesthesia. One of the major factors currently leading our patients away from END-DCR is general anesthesia. In the past few years, we have evolved a simplified local anesthesia technique which utilizes the minimum steps required to achieve excellent pain control and hemostasis without significant discomfort during application of the anesthetic either subcutaneously or in the nose. 


\section{Materials and methods}

No institutional review board approval was required before starting this study because, at the time of writing the protocol, the ethics committee of our university was not yet established. The study population was drawn from the practice of a single oculoplastic surgeon (HAT). The charts of 448 patients in two tertiary referral ophthalmology centers who consented to EXT-DCR surgery under local anesthesia from September 2006 to January 2011 were retrospectively reviewed. Local anesthesia was recommended for all patients older than 16 years. Patients were advised against local anesthesia if they reported previous allergies to lidocaine, epinephrine, or oxymetazoline, or were foreigners whose native language or Arabic dialect was alien to the operating room staff and would make communication impossible during the procedure.

No packing of the nose with pledgets or ribbon gauze was done in any patient. Local topical nasal anesthesia was achieved as follows. We discarded $17 \mathrm{~mL}$ from a $20 \mathrm{~mL}$ bottle of oxymetazoline hydrochloride $0.05 \%$ spray, and replaced them with $3 \mathrm{~mL}$ of lidocaine $2 \%(20 \mathrm{mg} / \mathrm{mL})$, yielding a final concentration of $0.025 \%$ oxymetazoline and $1 \%$ lidocaine $(3 \mathrm{~mL}$ oxymetazoline hydrochloride and $3 \mathrm{~mL}$ lidocaine). This oxymetazoline/lidocaine mixture contained a total of $60 \mathrm{mg}$ lidocaine. We trained a staff nurse to apply a few puffs, every 3-4 minutes, for 90 minutes prior to surgery in the preoperative waiting room outside the operating theater with the patient in the sitting position and the head tilted slightly upwards. The nurse was instructed to alert the anesthesiologist immediately if aspiration developed due to the local anesthetic. We routinely instructed our patients not to swallow and to immediately spit out whatever was left over in the oropharynx after each puff. In bilateral patients, we discarded $14 \mathrm{~mL}$ from the oxymetazoline bottle and replaced them with double the amount of lidocaine $(6 \mathrm{~mL})$. No sedation was used, enabling patients to control their airways themselves to reduce further the risk of aspiration. As we became more experienced with the technique, the patients were instructed to apply the puffs themselves 60 minutes prior to the procedure.

Patients were later admitted to the operating theater and topical local anesthetic eye drops (oxybuprocaine $\mathrm{HCl} 0.4 \%$ ) were instilled in the eye to be operated on. A cardiorespiratory monitor was placed and routine oxygen was administered. Any residual unused oxybuprocaine/lidocaine mixture was sprayed into the nose at this point before the patient was sedated.

Local anesthetic injection was undertaken with or without sedation according to the discretion of the anesthesiologist.
When needed, conscious sedation was achieved using a single bolus injection of propofol $0.5-1 \mathrm{mg} / \mathrm{kg}$ until the patient reached and maintained sedation level 3 or 4 on the original Wilson sedation scale. ${ }^{3}$

Local infiltration anesthesia consisted of a deep subcutaneous injection (at the level of the periosteum) of a $7 \mathrm{~mL}$ mixture of 2\% lidocaine and 1:100,000 epinephrine injected on the flat side of the nose beneath the incision site. Using a 25 gauge needle, a small medial peribulbar injection between the caruncle and the medial canthal tendon was also given ( $3 \mathrm{~mL}, 2 \%$ lidocaine without epinephrine evenly distributed at a variable depth of about 5-10 $\mathrm{mm}$ ). Once the injections were concluded, the patient was gently awakened, except in rare situations where continuous heavy sedation during the procedure was deemed necessary. In such cases, a propofol infusion of $0.05-0.1 \mathrm{mg} / \mathrm{kg}$ per minute was administered intravenously according to the age of the patient and depth of sedation required. When performing bilateral simultaneous surgery, the anesthesiologist injected the local anesthetic into both eyes simultaneously. Electrocardiography, heart rate, blood oxygen saturation, and noninvasive arterial blood pressure were monitored in all patients throughout the procedure.

The procedure was started 10 minutes after conclusion of the anesthetic injections and was performed as follows: a curved eyelid incision was made, starting $10 \mathrm{~mm}$ medial to the medial canthus slightly below the level of the medial canthal tendon, into the first lower eyelid crease and was continued straight to the bone. The periosteum and the underlying lacrimal sac were dissected off the bone. A rhinostomy approximately $20 \times 20 \mathrm{~mm}$ in diameter was performed using a Kerrison punch. A U-shaped incision was made in the nasal mucosa to create an anterior flap. The lacrimal sac was vertically incised with a number 11 scalpel blade, and a large anterior flap was created followed by excision of the residual posterior flap, the nasolacrimal duct, and the fundus of the sac. The internal punctum was routinely inspected, and later the lacrimal sac flap was sutured to the nasal mucosal flap with a continuous 6-0 polyglactin 910 suture. A bicanalicular silicone stent (lacrimal intubation set, Eagle Labs, Rancho Cucamonga, CA, USA) was passed and tied in the nasal cavity with several knots. Finally, the skin was sutured with interrupted 6-0 polyglactin 910 sutures.

\section{Results}

We retrospectively reviewed the charts of 448 patients who underwent 480 EXT-DCR procedures with our simplified local anesthesia technique (32 patients underwent 
bilateral simultaneous surgery). There were 371 females $(82.5 \%)$ and 77 males $(17.5 \%)$, ranging in age from 16 to 93 (48.2) years. Duration of surgery was recorded in 179 files and the mean was 16 minutes per eye. Total duration of stay inside the operating theater was recorded in the last 89 unilateral EXT-DCR patients and averaged 38 (range 30-42) minutes.

No sedation was required in 128 patients during the local anesthesia injection, while 320 patients received sedation during injection of the local anesthetic. A total of 436 patients did not need any sedation during the procedure itself. Heavy sedation (Wilson sedation scale 5) was essential in eight patients because they were severely anxious $(n=5)$ or persistently complained of intolerable pain $(n=3)$. One of these patients was a bilateral EXT-DCR patient. Four patients could not tolerate the procedure under local anesthesia and were converted to general anesthesia. Again, two of them were bilateral patients.

Additional local anesthetic was required in four patients. In two of these, the reason cited in the chart was that no medial peribulbar block was injected by the anesthesiologist. The other two patients were undergoing bilateral EXT-DCR, and considerable pain developed while operating on the second eye. Supplemental local anesthetic injection promptly solved the problem in all four patients. No untoward cardiovascular or central nervous system side effects were noted in any patient. Neither was any case of globe perforation recorded. More importantly, aspiration from the topical nasal local anesthesia was not encountered in any patient.

Intraoperative bleeding was very low, except in one patient. Severe postoperative epistaxis was observed in four patients, all of whom were managed successfully with a polyvinyl acetyl sponge (Meroce ${ }^{\circledR}$ nasal packing, Medtronic, Minneapolis, MN, USA). Temporary anosmia developed in one patient undergoing bilateral EXT-DCR. The patient started complaining of anosmia immediately after discharge from hospital. Computed tomography was ordered one week later, and ruled out the presence of infection or hematoma. The anosmia resolved spontaneously 2 weeks after surgery.

Success was defined as the ability to complete the procedure successfully without conversion to general anesthesia, without supplemental local anesthesia injections, and without resorting to heavy sedation. Using these criteria, the procedure was completed successfully in $432 / 448$ patients (96.4\%). The success rate for patients undergoing bilateral EXT-DCR was 84\% (27/32).

\section{Discussion}

In any surgical procedure, there is usually a smoother recovery, earlier discharge, reduced costs, and fewer anesthetic complications with local anesthesia than with general anesthesia. ${ }^{4,5}$ In ophthalmology, the growing trend towards local anesthesia has reportedly reduced the rate of surgical cancellations. ${ }^{6}$ Needless to say, apprehension and anxiety can be significant problems and a barrier to local anesthesia, but good patient rapport and a thorough explanation of the procedure may help prevent these problems.

Cocaine is traditionally considered the local anesthetic of choice for the nasal mucosa in dacryocystorhinostomy because it is the only local anesthetic with vasoconstrictor properties; ${ }^{7}$ however, we favor an oxymetazoline/lidocaine combination over cocaine because cocaine is not licensed for any medical use in Egypt and does not even have a schedule status in the country. Moreover, in previous studies, an oxymetazoline/lidocaine combination was found to offer adequate nasal anesthesia and blood loss compared with commercial cocaine..$^{7,8}$

To the best of our knowledge, all previous publications dealing with local anesthesia for dacryocystorhinostomy utilized nasal packing to achieve hemostasis and pain control. ${ }^{4,8-14}$ An exception to this seemingly universal rule was reported only once in the Iranian literature, where the authors oddly reported minimal blood loss and no pain without the use of any nasal decongestant or anesthetic at all, either in the form of nasal packing or in the form of spray. ${ }^{15}$

We have not used any form of nasal packing for the past 5 years, and instead rely on spraying the nose with anesthetic and decongestant prior to the procedure, with excellent hemostasis and pain control. Although not part of the present case series, we have occasionally tried to avoid packing the nose with gauze since 2001. Initially, we used a separate oxymetazoline $0.05 \%$ aerosolizing spray and a lidocaine pump spray (10\%), applied each type of spray ten times, a few minutes apart about half an hour prior to surgery, inside the operating room. These patients universally complained of an unpleasant taste, gagging, and throat discomfort from the large volume of lidocaine reaching the oropharynx. Because EXT-DCR is performed relatively anteriorly in the nasal cavity, and because the nozzle of the commercially available lidocaine pump spray is unnecessarily long for our purpose, and is probably the culprit behind throat discomfort, we have modified the technique by mixing oxymetazoline and lidocaine in the oxymetazoline spray bottle with its considerably shorter nozzle. No adverse effects have been reported 
in the literature ${ }^{7}$ or in our patients from mixing these drugs, and throat discomfort is no longer a concern.

Previous studies have confirmed that the use of lidocaine spray with epinephrine provides adequate nasal anesthesia and decongestion for rhinologic procedures other than EXT-DCR, ${ }^{16}$ but cotton pledgets are required to provide maximal anesthesia and turbinate shrinkage. ${ }^{17}$ The quintessential question is whether this technique would be sufficient for END-DCR where maximal anesthesia and hemostasis are prerequisites to successfully carry out the procedure under local anesthesia. Conventional wisdom would argue against its effectiveness, although a separate study is required to test this hypothesis.

The use of $1 \%$ inhalational lidocaine instead of the commercially available $10 \%$ concentration is not new. In a previous study comparing different concentrations for inhalational lidocaine, it was concluded that the $1 \%$ concentration may be as effective as higher concentrations, with less toxicity. ${ }^{18}$

The concentration of oxymetazoline used in this study was also low $(0.025 \%)$ which is half the concentration of commercially available oxymetazoline, and although no documentation of the amount of blood loss was attempted in our study, hemostasis was excellent, the nasal volume was roomy, and the surgical space was adequate. The basis of our success with a lower concentration of oxymetazoline could be found in a previous study comparing $0.25 \mathrm{mg} / \mathrm{mL}$ oxymetazoline versus $0.5 \mathrm{mg} / \mathrm{mL}$ oxymetazoline which surprisingly found no statistically significant advantage for using the higher concentration and called for a reduction of the oxymetazoline dose in over-the-counter medications based on a hypothesized ceiling effect which is present at a concentration of $0.025 \% .{ }^{19}$ Alternatively, the sheer large volume of oxymetazoline that was used in the current study ( $3 \mathrm{~mL}$ ) may have compensated for the lower concentration used $(0.025 \%)$.

It is important to note that five of the 16 "failures" in the study were patients undergoing bilateral EXT-DCR and, in all of them, pain was intolerable by the time we started operating on the second eye. The lower success rate in bilateral patients argues against using this minimalist technique in this particular cohort of patients. An obvious way out would have been to use a longer-acting anesthetic like bupivacaine, and in fact it would seem rather antithetical that we continued using a shorter-acting anesthetic in all our subsequent bilateral patients. The reason why we favored lidocaine over long-acting amide anesthetics like bupivacaine is that the percentage of unionized active lidocaine is higher than bupivacaine at a $\mathrm{pH}$ of 7.4 (25\% versus $15 \%) .{ }^{20}$ In an infected culture-positive milieu, a common situation encountered with dacryocystorhinostomy patients, ${ }^{21,22}$ tissues tend to be more acidic than usual, and because the $\mathrm{pH}$ is reduced, the fraction of the unionized active drug is also reduced, thereby decreasing the potency of the anesthetic, because the ionized form of any anesthetic cannot readily penetrate cell membranes to exert its action. ${ }^{23}$ An alternative approach to improve the success in bilateral EXT-DCR may be to defer anesthetizing the second eye until after surgery on the first eye is finished, but this would entail rescrubbing the patient after the anesthesiologist is finished with the injections, with an inevitable attendant delay in the operating room.

Techniques used for infiltration of local anesthesia in EXT-DCR run the gamut from elegant yet complicated techniques to oversimplified single-injection procedures. ${ }^{4,8-15} \mathrm{On}$ the humbler end of the spectrum, one author used a single subcutaneous injection and omitted the medial peribulbar injection altogether and reported only mild patient discomfort during osteotomy. ${ }^{10}$ In two of our patients, the anesthesiologist missed giving the medial peribulbar injection and used a single subcutaneous injection instead. It was not clear in the charts whether or not this was an inadvertent error, but patients were not comfortable during the osteotomy, especially with the initial outfracturing of bone in the lacrimal fossa and additional local anesthetic had to be used. Our limited experience with these two patients belies the favorable response that the aforementioned author has experienced using his technique. In this regard, our technique is only slightly dissimilar to the one described by Caesar and $\mathrm{McNab}$, who used three injections with the exception of the infraorbital block which we have omitted. ${ }^{9}$ We believe that limiting the number of injections to two is a tradeoff decreasing discomfort from complicated injections while at the same time eliminating pain during the procedure.

One of the major drawbacks of the study is that no pain scoring system was used to assess overall patient discomfort with the injection technique or pain intensity during the procedure itself. Another caveat is that pain sensation in the nasal mucosa was not measured quantitatively with commercially available kits to gauge sensation threshold and pain perception. Further, we did not delve into the final outcome of dacryocystorhinostomy under local anesthesia versus general anesthesia, or compare our simplified local anesthesia technique with other published local anesthesia methods. Again, and because of the retrospective nature of this research, we did not compare the intraoperative and postoperative complications with those of EXT-DCR under general anesthesia, or quantify and compare the amount of 
blood loss under local anesthesia versus general anesthesia. We also failed to measure the arterial plasma concentrations of lidocaine to quantitatively assess our alleged lack of toxicity at the low concentration used. Finally, the repeated applications of oxymetazoline/lidocaine puffs over an extended period of time ( 90 minutes) may be cumbersome for some patients.

Simply using "successful completion" of the procedure alone as the main outcome measure may not attest to the difficulties encountered until a certain procedure is completed, and indeed it may not be judicious to use the operating surgeon as a reference to reflect on the plausibility of a suggested surgical or anesthetic maneuver. Therefore, our primary outcome measure was not merely determined by "successful completion" of the procedure, but was expanded to ensure that heavy sedation, conversion to general anesthesia, and additional lidocaine supplements (injectable or inhalational) were not required during the procedure to complement the original anesthetic, and with these parameters it was a success. Our definition of success excluded patients to whom heavy sedation was administered because, in addition to having its own set of side effects, we believe that implementing heavy sedation without airway control in a procedure where intraoperative blood loss could be significant may have disastrous consequences. ${ }^{24}$

Although our enthusiasm for the technique should be tempered by the lack of pain scores and the lower success rate in bilateral patients, our simplified anesthetic approach has evolved over the past 10 years in such a reliable manner that it has become a routine everyday practice in several ophthalmology centers around the country, except in pediatric DCR. We propose that our technique confers the following advantages: EXT-DCR and the antecedent anesthetic injections can be routinely performed under minimal or no sedation; it is well tolerated even in younger patients; obviates the need for multiple injections and complicated nerve blocks; abolishes the pain endured by patients during application and removal of nasal packing when performed without sedation; and eliminates the potential for aspiration when nasal packing is performed in sedated patients. ${ }^{25,26}$ Further, it offers significant reduction of the time spent by the patient inside the operating room. In a busy clinical practice setting, rapid patient turnover and avoidance of general anesthesia have clear financial merits, and help reduce patient concerns over anesthetic risks. Based on the results of this pilot study, where the results were interpreted entirely subjectively, a separate study may be needed to assess objectively and quantitatively whether the discomfort caused by application of nasal pledgets soaked with local anesthetics and decongestants in the nose outweighs the discomfort caused by our technique.

\section{Disclosure}

This paper was presented in part at the European Society of Ophthalmic Plastic and Reconstructive Surgery annual meeting, Lucerne, Switzerland, September 19-21, 2008. This study did not receive financial support. The authors have no proprietary or commercial interest in any materials discussed in this paper.

\section{References}

1. Ciftci F, Dinc UA, Ozturk V. The importance of lacrimal diaphragm and periosteum suturation in external dacryocystorhinostomy. Ophthal Plast Reconstr Surg. 2010;26:254-258.

2. Heindl LM, Junemann A, Holbach LM. A clinicopathologic study of nasal mucosa in 350 patients with external dacryocystorhinostomy. Orbit. 2009;28:7-11.

3. Wilson E, David A, Mackenzie N, Grant IS. Sedation during spinal anesthesia: comparison of propofol and midazolam. Br J Anaesth. 1990;64:48-52.

4. Ciftci F, Pocan S, Karadayi K, Gulecek O. Local versus general anesthesia for external dacryocystorhinostomy in young patients. Ophthal Plast Reconstr Surg. 2005;21:201-206.

5. Nordin P, Zetterström H, Gunnarsson U, Nilsson E. Local, regional, or general anaesthesia in groin hernia repair: multicentre randomised trial. Lancet. 2003;362:853-858.

6. Hussain R, Matare T, Zambarakji H. National survey of day-case vitreoretinal surgery in the United Kingdom. Eur J Ophthalmol. 2011;21:183-188.

7. Noorily AD, Noorily SH, Otto RA. Cocaine, lidocaine, tetracaine: which is best for topical nasal anesthesia? Anesth Analg. 1995;81:724-727.

8. Meyer DR. Comparison of oxymetazoline and lidocaine versus cocaine for outpatient dacryocystorhinostomy. Ophthalmic Plast Reconstr Surg. 2000;16:201-205.

9. Caesar RH, McNab AA. External dacryocystorhinostomy and local anesthesia: technique to measure minimized blood loss. Ophthal Plast Reconstr Surg. 2004;20:57-59.

10. Maheshwari R. Single-prick infiltration anesthesia for external dacryocystorhinostomy. Orbit. 2008;27:79-82.

11. Howden J, McCluskey P, O’Sullivan G, Ghabrial R. Assisted local anaesthesia for endoscopic dacryocystorhinostomy. Clin Experiment Ophthalmol. 2007;35:256-261.

12. Riad W, Chaudhry IA. Anaesthesia for dacryocystorhinostomy. Curr Anaesth Crit Care. 2010;21:180-183.

13. McNab A, Simmie RJ. Effectiveness of local anaesthesia for external dacryocystorhinostomy. Clin Experiment Ophthalmol. 2002;30:270-272.

14. Fanning GL. Local anaesthesia for dacryocystorhinostomy. Curr Anaesth Crit Care. 2000;11:306-309.

15. Kasaee A, Ghahari E, Tabatabaie S. External dacryocystorhinostomy: local versus general anesthesia. Iran J Ophthalmol. 2010;22:27-30.

16. Kasemsuwan L, Griffiths MV. Lignocaine with adrenaline: is it as effective as cocaine in rhinological practice? Clin Otolaryngol Allied Sci. 1996;21:127-129.

17. Lee YC, Wang CP. Cotton pledget method for nasal decongestive anesthesia prior to transnasal endoscopy. Am J Gastroenterol. 2008;103: 3212-3213.

18. Mainland PA, Kong AS, Chung DC, Chan CH, Lai CK. Absorption of lidocaine during aspiration anesthesia of the airway. J Clin Anesth. 2001;13:440-446. 
19. Hummel T, Rothbauer C, Pauli E, Kobal G. Effects of the nasal decongestant oxymetazoline on human olfactory and intranasal trigeminal function in acute rhinitis. Eur J Clin Pharmacol. 1998;54:521-528.

20. Becker DE, Reed DL. Essentials of local anesthetic pharmacology. Anesth Prog. 2006;53:98-109.

21. DeAngelis D, Hurwitz J, Mazzulli T. The role of bacteriologic infection in the etiology of nasolacrimal duct obstruction. Can J Ophthalmol. 2001;36:134-139.

22. Bharathi MJ, Ramakrishnan R, Maneksha V, Shivakumar C, Nithya V, Mittal S. Comparative bacteriology of acute and chronic dacryocystitis. Eye. 2008;22:953-960.
23. Bahl R. Local anesthesia in dentistry. Anesth Prog. 2004;51:138-142.

24. Minocha A, Asan RS. Conscious sedation peals and perils. Dig Dis Sci. 1998;43:1835-1844.

25. Koudounarakis E, Chatzakis N, Papadakis I, Panagiotaki I, Velegrakis G. Nasal packing aspiration in a patient with Alzheimer's disease: a rare complication. Int J Gen Med. 2012;5:643-645.

26. Eipe N, Choudhrie A. Nasal pack causing upper airway obstruction. Anesth Analg. 2005;100:1861.
Clinical Ophthalmology

\section{Publish your work in this journal}

Clinical Ophthalmology is an international, peer-reviewed journal covering all subspecialties within ophthalmology. Key topics include: Optometry; Visual science; Pharmacology and drug therapy in eye diseases; Basic Sciences; Primary and Secondary eye care; Patient Safety and Quality of Care Improvements. This journal is indexed on

\section{Dovepress}

PubMed Central and CAS, and is the official journal of The Society of Clinical Ophthalmology (SCO). The manuscript management system is completely online and includes a very quick and fair peer-review system, which is all easy to use. Visit http://www.dovepress.com/ testimonials.php to read real quotes from published authors. 